\title{
Fiber-Optic Fabry-Perot Hydrogen Sensor Coated With Pd-Y Film
}

\author{
Caibin YU ${ }^{1}$, Li LIU ${ }^{1}$, Xiaoxiao CHEN ${ }^{2}$, Qunfeng $\mathrm{LIU}^{1}$, and Yuan $\mathrm{GONG}^{1 *}$ \\ ${ }^{1}$ Key Laboratory of Optical Fiber Sensing and Communications (Ministry of Education of China), University of \\ Electronic Science and Technology of China, Chengdu, 611731, China \\ ${ }^{2}$ National Center of Quality Inspection for Sensors, National Institute of Measurement and Testing Technology, Chengdu, \\ 610021, China
}

${ }^{*}$ Corresponding author: Yuan GONG $\quad$ E-mail: gong.yuan@163.com

\begin{abstract}
A fiber-optic Fabry-Perot hydrogen sensor was developed by measuring the fringe contrast changes at different hydrogen concentrations. The experimental results indicated that the sensing performance with the Pd-Y film was better than that with the Pd film. A fringe contrast with a decrease of $0.5 \mathrm{~dB}$ was detected with a hydrogen concentration change from $0 \%$ to $5.5 \%$. The temperature response of the sensor was also measured.
\end{abstract}

Keywords: Optical fiber sensor, hydrogen sensor, Pd-Y film, fringe contrast, temperature characteristics

Citation: Caibin YU, Li LIU, Xiaoxiao CHEN, Qunfeng LIU, and Yuan GONG, "Fiber-Optic Fabry-Perot Hydrogen Sensor Coated With Pd-Y Film," Photonic Sensors, 2015, 5(2): 142-145.

\section{Introduction}

Clean and renewable energy has attracted much attention, among which hydrogen is one of the most promising ways. Hydrogen has many advantages, such as cleanness, reproducibility, lightness, and big heat capacity. However, it is very dangerous for us to store or transport hydrogen because of its wide explosion concentration range and low ignition. Various hydrogen sensors have been investigated and reported in literatures so far, based on semiconductor, electrochemical $[1,2]$ and fiber optic schemes [3-6]. Semiconductor and electrochemical sensors work based on their electrical characteristics. They may produce sparks and cause an explosion when the hydrogen concentration exceeds the lowest explosive limit. The optical fiber hydrogen sensor is inherently safe.
Palladium (Pd) has been widely used in hydrogen sensors because of its high sensitivity and selective affinity [7-10]. The further Pd-Y film was used for hydrogen sensing [11]. In this paper, a novel hydrogen sensor is proposed and demonstrated based on the graded-index multimode fiber (GI-MMF) Fabry-Perot (FP) structure [12] coated with a Pd-Y film. The sensing performance is measured.

\section{Experimental setup}

The sensor consists of a GI-MMF FP structure and a Pd-Y film coated at the fiber end, as shown in Fig. 1.

The fabrication process of the FP structure was described in detail in [12]. The sensor with this structure has the advantages of high fringe contrast, low cost, high sensitivity, and easy fabrication, and

Received: 21 December 2014 / Revised version: 12 January 2015

(C) The Author(s) 2015. This article is published with open access at Springerlink.com

DOI: $10.1007 / \mathrm{s} 13320-015-0237-0$

Article type: Regular 
it can measure refractive index and temperature simultaneously. There are three reflective interfaces in this sensor, including the single mode fiber (SMF) end, the air-gap, and the GI-MMF end, respectively.

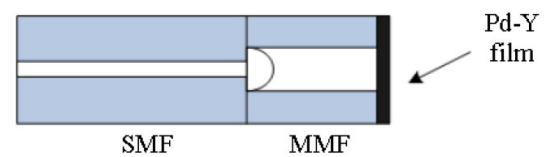

Fig. 1 Structure of the fiber-optic Faber-Perot (FFP) hydrogen sensor.

The Pd-Y film absorbs hydrogen, and PdH-Y is produced, leading to the changes in both optical loss and the refractive index of the film. Pd-Y was proved to be an ideal sensitive material for a hydrogen sensor [11]. The optical fiber sensor measures refractive index changes based on the interaction between the hydrogen and Pd-Y film, which induces the change in the refractive index of the Pd-Y film which is coated on the optical GI-MMF fiber end. Further, the fringe contrast of the reflective spectrum of the FP sensor will change. Meanwhile, the temperature can be measured according to the wavelength shift of the spectrum.

The experimental setup is schematically shown in Fig. 2. The wavelength-swept laser based optical spectrum analyzer was used to measure the reflective spectrum of the sensor. The range of the input spectrum was tunable from $1510 \mathrm{~nm}$ to $1590 \mathrm{~nm}$. The hydrogen sensor was placed in a chamber with a fixed volume. The volume was about $600 \mathrm{~mL}$. Different hydrogen concentrations were controlled by means of flow valve control, ranging from $0 \%$ to $6 \%$. A set of data were recorded

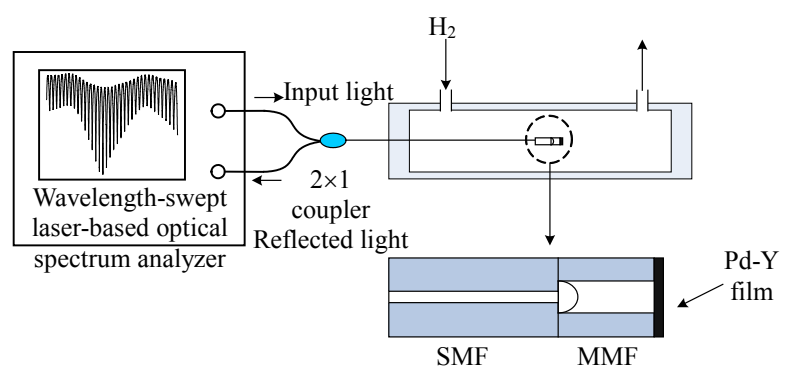

Fig. 2 Schematic setup of the FFP sensor used to detect hydrogen gas. every half hour in order to let the film and hydrogen have a sufficient reaction.

We took the sensor out of the chamber and exposed it to air for sufficient time in order to let the PdH-Y transform into Pd-Y completely. Then the temperature response of the sensor was measured.

\section{Results and characteristics}

The reflective spectra of the FFP sensor with the Pd-Y film and with the Pd film in different hydrogen concentrations are shown in Figs. 3(a) and 3(b), respectively. In the first experiment with the Pd-Y film, we tested the hydrogen volumetric concentration at $0 \%, 1.5 \%, 3.17 \%, 4.8 \%$, and $5.7 \%$, respectively. It is indicated by the experimental results that the interference fringe contrast decreases as the hydrogen concentration increases and the wavelength shift is small. The maximum fringe contrast obtained in this experiment was about $22.75 \mathrm{~dB}$.

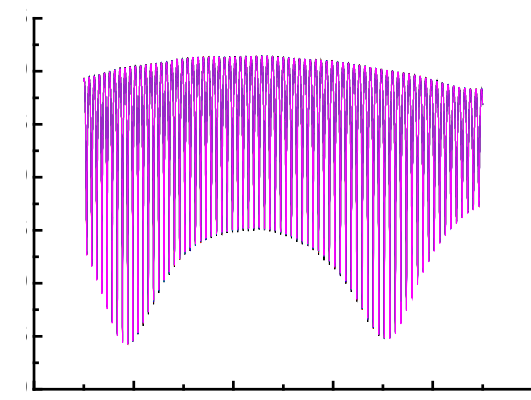

(a)

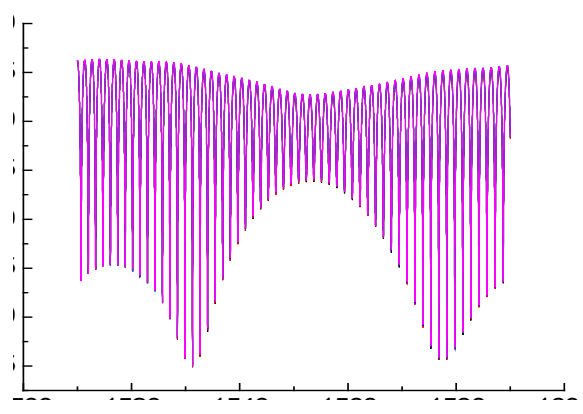

(b)

Fig. 3 Reflective spectra of the FFP sensor with the (a) Pd-Y film and (b) Pd film.

In order to make a comparison, we used another 
FFP sensor coated with the Pd film instead of the Pd-Y film to repeat the experiment with the hydrogen concentration of $0 \%, 0.83 \%, 1.67 \%$, $2.50 \%$, and $3.33 \%$, respectively. The maximum fringe contrast of the point we chose in this experiment was about $26.41 \mathrm{~dB}$.

Figure 4 shows that with an increase in the hydrogen concentration, the fringe contrast of the FFP sensor decreases. It provides a basis for us to be able to detect the hydrogen concentration. A fringe contrast of $0.5 \mathrm{~dB}$ was detected for the FFP sensor with the Pd-Y film when the hydrogen concentration changed from $0 \%$ to $5.5 \%$, while of $0.2 \mathrm{~dB}$ for the FFP sensor with the Pd film when the concentration changed from $0 \%$ to $3.3 \%$. The FFP sensor with the $\mathrm{Pd}-\mathrm{Y}$ film is more suitable for a hydrogen sensor than that with the Pd film, in both the sensitivity and linearity.

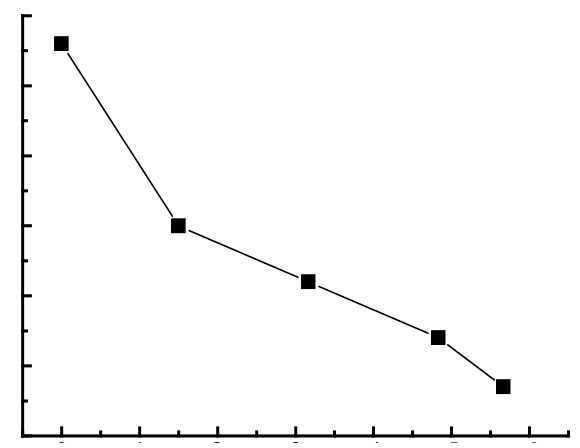

(a)

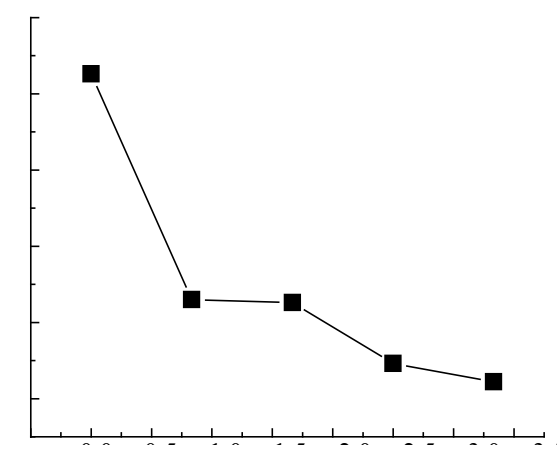

(b)

Fig. 4 Fringe contrast versus hydrogen concentration by the FFP sensor with the (a) Pd-Y film and (b) Pd film.

The scanning electron microscopy (SEM) images of the Pd-Y film before and after hydrogen sensing are shown in Fig. 5. The Pd-Y film has the good recovery property and can be used for multiple times for hydrogen sensing. In this aspect, the performance of the Pd film is not as good as the Pd-Y film [11].
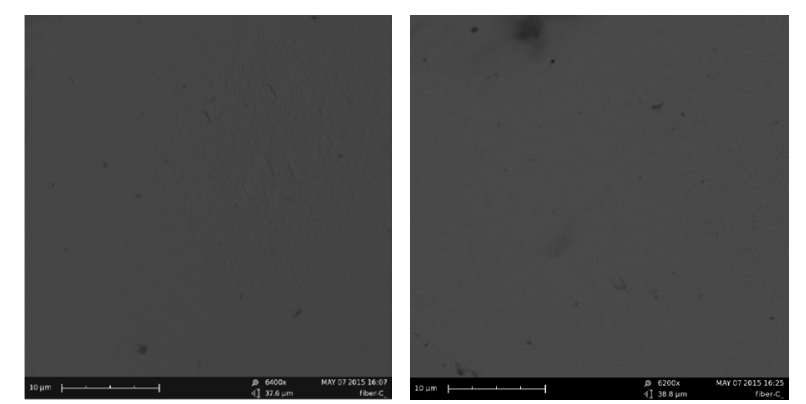

Fig. 5 SEM images of the Pd-Y film before (left) and after (right) hydrogen sensing.

The temperature responses of the FFP sensor are shown in Fig. 6. According to the previous research, the GI-MMF FFP sensor can measure the temperature and refractive index simultaneously with low cross talking. Temperature mainly influences the wavelength shift of the interference fringes. Good linearity is shown in Fig. 6. The fringe contrast change induced by the temperature variation is negligible. The temperature responses of the FFP sensor with the Pd-Y film and Pd film are almost the same.

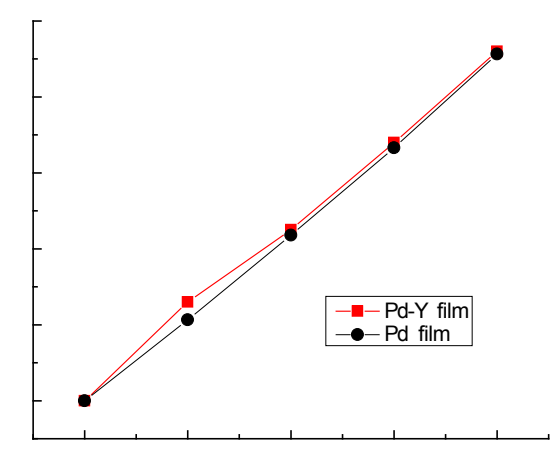

Fig. 6 Wavelength shift of the FFP sensor as a function of temperature.

\section{Conclusions}

An optical fiber hydrogen sensor based on the graded-index multimode fiber Fabry-Perot 
interferometer coated with the Pd-Y film is proposed and developed. The hydrogen concentration and temperature can be determined by measuring the fringe contrast and wavelength shift of the reflective spectrum, respectively. Compared with the Pd film, the Pd-Y film has advantages of high sensitivity and linearity.

\section{Acknowledgment}

This work is supported by the National Natural Science Foundation of China (61107073, 61107072, and 61290312), Fundamental Research Funds for the Central Universities (ZYGX2011J002), Research Fund for the Doctoral Program of Higher Education of China (20110185120020), Program for Changjiang Scholars and Innovative Research Team in University (PCSIRT, IRT1218), and the 111 Project (B14039).

Open Access This article is distributed under the terms of the Creative Commons Attribution License which permits any use, distribution, and reproduction in any medium, provided the original author(s) and source are credited.

\section{References}

[1] L. Boon-brett, J. Bousek, P. Castello, O. Salyk, F. Harskamp, L. Aldea, et al., "Reliability of commercially available hydrogen sensors for detection of hydrogen at critical concentrations: part I - testing facility and methodologies," International Journal of Hydrogen Energy, 2008, 33(24): 7648-7657.

[2] L. Boon-brett, J. Bousek, and P. Moretto, "Reliability of commercially available hydrogen sensors for detection of hydrogen at critical concentrations: part II - selected sensor test results," International Journal of Hydrogen Energy, 2009, 34(1): 562-571.

[3] C. Christophe, D. Marc, L. Driss, and M. Patrice, "Hybrid fiber gratings coated with a catalytic sensitive layer for hydrogen sensing in air," Optics Express, 2008, 16(21): 16854-16859.

[4] X. Bevenot, A. Trouillet, C. Veillas, H. Gagnaire, and M. Clement, "Surface plasmon resonance hydrogen sensor using an optical fibre," Measurement Science and Technology, 2002, 13(1): 118-124.

[5] S. Sumida, S. Okazaki, S. Asakura, H. Nakagawa, H. Murayama, and T. Hasegawa, "Distributed hydrogen determination with fiber-optic sensor," Sensors and Actuators B: Chemical, 2005, 108(1-2): 508-514.

[6] C. Caucheteur, M. Debliquy, D. Lahem, and P. Megret, "Catalytic fiber Bragg grating sensor for hydrogen leak detection in air," IEEE Photonics Technology Letters, 2008, 20(2): 96-98.

[7] M. Buric, T. Chen, M. Maklad, P. R. Swinehart, and K. P. Chen, "Multiplexable low-temperature fiber Bragg grating hydrogen sensors," IEEE Photonics Technology Letters, 2009, 21(21): 1594-1596.

[8] M. Wang, M. Yang, J. Cheng, J. Dai, M. Yang, and D. Wang, "Femtosecond laser fabricated micro Mach-Zehnder interferometer with Pd film as sensing materials for hydrogen sensing," Optics Letters, 2012, 37(11): 1940-1942.

[9] M. David, L. Donato, M. E. Dalia, and V. Joel, "Optical microfibers decorated with PdAu nanoparticles for fast hydrogen sensing," Sensors and Actuators B: Chemical, 2010, 151(1): 219-222.

[10] M. David, L. Donato, and M. Dalia, "Fast response fiber optic hydrogen sensor based on palladium and gold nano-layers," Sensors and Actuators B: Chemical, 2009, 136(2): 562-566.

[11] M. Yang and J. Dai, "Fiber optic hydrogen sensors: a review," Photonic Sensors, 2014, 4(4): 300-324.

[12] Y. Gong, T. Zhao, Y. Rao, Y. Wu, and Y. Guo, "A ray-transfer-matrix model for hybrid fiber Fabry-Perot sensor based on graded-index multimode fiber," Optics Express, 2010, 18(15): 15844-15852. 